\title{
Fast electrophoresis in conventional capillaries by employing a rapid injection device and contactless conductivity detection
}

Received: 26 October 2004/ Revised: 21 December 2004/ Accepted: 22 December 2004 / Published online: 17 February 2005

(C) Springer-Verlag 2005

\begin{abstract}
A purpose-made set-up featuring an automated fast injector allowed the easy optimization of the injected amount and the adjustment of the separation length of conventional capillaries from a minimum of $5 \mathrm{~cm}$ upward. It was found that a compromise in capillary length for separation efficiency and analysis time also has to take into account the injected amount, which in turn affects the sensitivity and hence the detection limit. The versatility of the system was demonstrated by the analysis of the major cations and anions in natural water samples in less than $1 \mathrm{~min}$, the concurrent determination of a mixture of amino acids and carbohydrates in $160 \mathrm{~s}$, and of three active ingredients in a pharmaceutical preparation in $40 \mathrm{~s}$. Plate numbers were typically around 50,000 and detection limits down to $1 \mu \mathrm{M}$ could be achieved.
\end{abstract}

Keywords Capillary electrophoresis - Fast separation · Automated injection - Contactless conductivity detection

\section{Introduction}

The most common detection method in capillary electrophoresis (CE) is UV absorption; however, indirect techniques with limited sensitivity have to be used for the non-absorbing metal ions, inorganic anions, and aliphatic organic ions. Fluorescence, which has high sensitivity, is largely restricted to species which can be readily derivatized to render them fluorescent. An important alternative method was introduced in 1998 by Zemann et al. and do Lago et al. in the form of contactless conductivity detection (CCD) $[1,2]$. The method may be considered universal in $\mathrm{CE}$ as inherently all ions

A. Rainelli · P. C. Hauser $(\square)$

Department of Chemistry, University of Basel, Spitalstrasse 51, 4004 Basel, Switzerland

E-mail: peter.hauser@unibas.ch can be sensed. Furthermore, this electrochemical detection method has the robustness of optical detection, since the electrodes cannot deteriorate (because they are not in contact with the solution) and the fact that the entire detection volume is probed and not merely a twodimensional interface. For these reasons the method has attracted considerable attention and the topic has been reviewed several times [3-5]. Contactless conductivity detection has, for example, been demonstrated in CE for small inorganic cations (ammonia and alkali and alkaline earth ions) [6, 7], small inorganic anions (halides, oxo-anions) [1, 8, 9], carboxylic acids [10], sulfonates [11, 12], amines [11], organic anions and cations of pharmaceutical relevance [13], amino acids (without derivatization) [14, 15], carbohydrates (at pH 13) [16], and other species.

On microfabricated electrophoresis chips the need for alternative detection methods is pressing as UV absorption has not been successful because of the short optical path lengths available. Contactless conductivity detection has therefore also been implemented on this separation platform (e.g., see ref. [17]). The main advantage of these devices is the fast analysis times achieved. Separations in less than a minute are frequently reported for the chip format for analyses which would require 5-20 $\mathrm{min}$ in conventional $\mathrm{CE}$. The reason for the discrepancy is the length of the separation channel which is only a few centimeters on chips but typically $40 \mathrm{~cm}$ or more for conventional capillaries. It is not readily possible to emulate these fast separations in conventional $\mathrm{CE}$ as the commercial instruments are not designed to be used with very short capillary lengths. However, several researchers have demonstrated the utility of short conventional capillaries by employing UV detection on purpose-made apparatus or commercial instruments modified for injection from the detection end.

Capillaries of $7.5-\mathrm{cm}$ length have been used by Zemann for the separation of a range of inorganic and organic anions in less than $1 \mathrm{~min}$ [18], and Yang and coworkers showed the fast determination of inorganic 
anions in capillaries of 13-cm length [19-21]. Bjørnsdottir and Hansen [22] and Riekkola and coworkers [23] have demonstrated the determination of illicit drugs in 2 min by using capillaries of $8.5-$ and $23-\mathrm{cm}$ effective lengths, respectively. Li and coworkers [24] compared a conventional capillary of $14-\mathrm{cm}$ length with a microfabricated chip device and found that both systems gave a similar performance.

The aim of the project reported herein was to evaluate the combination of contactless conductivity detection with short conventional capillaries. We aimed to achieve fast separations without incurring the complications of microfabrication, while making use of the universality of the conductometric detection method. We expected that the injection time would be critical and in order to achieve fast and reproducible injections an automated system was employed.

\section{Experimental}

\section{Instrumentation}

The electrophoretic instrument was built in-house and consists of a fast injector device, a high-voltage supply, and a contactless conductivity detector. A representation of the CE system is given in Fig. 1. The injector is based on a design previously described by Hooker and Jorgenson [25] for coupling CE to HPLC. It consists of a clear polycarbonate disk of $4-\mathrm{cm}$ diameter and $1.2-\mathrm{cm}$ thickness with a cross-shaped channel of 1/16-in. diameter. At all four ends threads for 1/4-in. $\times 28$ type fittings were cut. Into one of the channels the separation capillary and the capillary which feeds the sample to the cross were fitted from opposite ends with the help of tubing sleeves of $395-\mu \mathrm{m}$ i.d. and 1/16-in. o.d. The distance between the two capillaries in the cross was ca. $0.5 \mathrm{~mm}$. To the other channel a PEEK-tubing with $0.5-\mathrm{mm}$ i.d. and 1/16-in. o.d. was fitted for feeding the buffer solution to the interface and opposite an electrically grounded piece of stainless steel tubing of $0.75-\mathrm{mm}$ i.d. and 1/16-in. o.d. was attached through which the solution flows to waste. All fittings, PEEK-tubings, and sleeves were obtained from Upchurch Scientific (Oak Harbor, WA, USA) and the fused silica capillaries were purchased from Polymicro Technologies (Phoenix, AZ,
USA; $15,25,50$, or $75-\mu \mathrm{m}$ i.d., $375-\mu \mathrm{m}$ o.d.). When the pinch valve (225P081-11, NResearch, West Caldwell, NJ, USA) is closed, the buffer flow generated by a syringe pump (Bee-Hive system from BAS, West Lafayette, IN, USA, fitted with a 1,000 Series Gastight, TTL-syringe from Hamilton, Bonaduz, Switzerland) is directed to the interface and the sample flows to waste. In the open position, the sample is passed to the interface while the buffer goes directly to waste.

Injection and separation voltages were produced by a high-voltage power supply (Spellman CZE 2000; Spellman Ltd., Pulborough, UK) at the detection end of the capillary which was placed in a perspex box for safety. Injection was effected by switching the valve to open in order to stop the buffer flowing through the cross and directing the sample to the interface. At the same time the high voltage was applied for a specific length of time to carry out an electrokinetic injection. The standards and samples were contained in the buffer solution in order to avoid any stacking effects. The valve and the high voltage were turned off simultaneously for $1 \mathrm{~s}$ to flush the interface with buffer and finally the separation voltage was turned on. The valve, pumps, and high voltage supply were controlled via a purpose-made interface with a program (LabVIEW, National Instruments, Austin, TX, USA) running on a notebook computer fitted with a multipurpose interface card (DAQCARD-6042E, National Instrument). The electropherograms were recorded with a MacLab/4e data acquisition system (AD Instruments, Castle Hill, Australia).

Detection was carried out with a high-voltage contactless conductometric detector similar to the one described previously and has a gap size of $1 \mathrm{~mm}$ unless stated otherwise $[6,11]$. The detector cell can be moved readily along the capillary as no window has to be created in the capillary coating for contactless conductivity detection. The shortest possible capillary length from the injection point to the detector cell is $5 \mathrm{~cm}$. Cell excitation was carried out at $720 \mathrm{kHz}$ and $30 \mathrm{~V}_{\mathrm{p}-\mathrm{p}}$. The pick-up amplifier used in the experiment was an OPA606 (Texas Instruments, Dallas, TX, USA) with a feedback resistor of $1 \mathrm{M} \Omega$ for the capillaries of 15,25 , and $50-\mu \mathrm{m}$ i.d. and $680 \mathrm{k} \Omega$ for the $75-\mu \mathrm{m}$ capillary. The detection limits are given for a signal-to-noise ratio of 3 .
Fig. 1 Schematic drawing of the experimental set-up

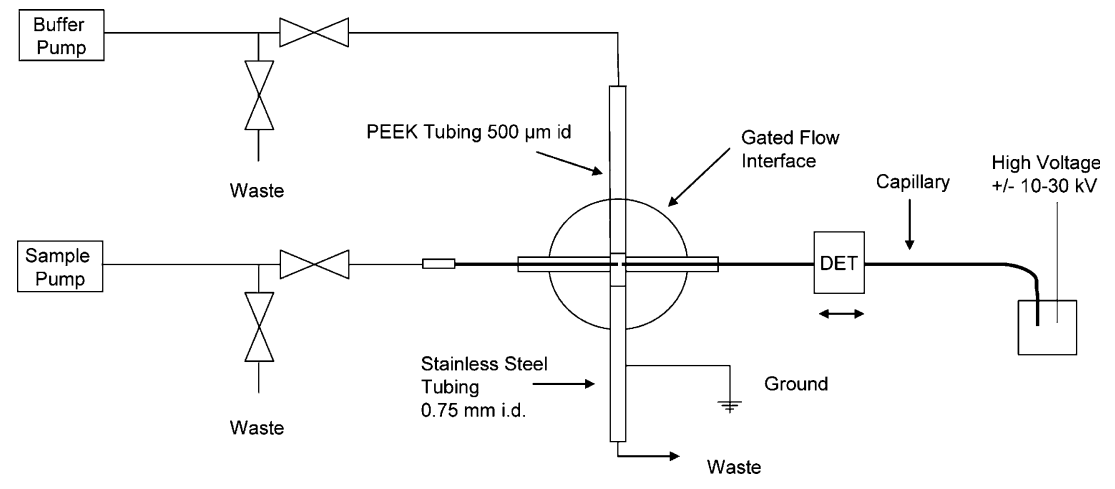


Reagents and methods

All chemicals were of analytical reagent grade. The inorganic salts, amino acids, and the saccharides were purchased from Fluka (Buchs, Switzerland). A $20 \mathrm{mM}$ 2-( $N$-morpholino)ethanesulfonic acid/histidine (MES/ His) buffer (pH 6.1) was used for the fundamental studies, the determination of inorganic cations and anions, and the separation of the pharmaceutical preparation. For anion analysis $0.00015 \%$ hexadimethrine bromide was added to the buffer for modification of the electroosmotic flow. Stock solutions of inorganic anions and cations were prepared from the potassium and chloride salts, respectively. The carbohydrates were separated in a buffer consisting of $10 \mathrm{mM} \mathrm{NaOH}$, $4.5 \mathrm{mM} \mathrm{Na}_{2} \mathrm{HPO}_{4}$, and $0.00015 \%$ hexadimethrine bromide, and the mixture of carbohydrates and amino acids in $10 \mathrm{mM} \mathrm{NaOH}, 4.5 \mathrm{mM} \mathrm{Na}_{2} \mathrm{HPO}_{4}$, and $1 \mathrm{mM}$ cetyltrimethylammonium bromide (CTAB). All solutions were diluted with ultra-pure water (Millipore, Bedford, MA, USA), degassed and filtered with $0.45-\mu \mathrm{m}$ nylon filters (BGB Analytik, Böckten, Switzerland).

\section{Results and discussion}

Electropherograms for the four inorganic cations (potassium, sodium, magnesium, and lithium) obtained in short capillaries of between 5 - and $20-\mathrm{cm}$ effective length from injection to detection are given in Fig. 2. Clearly, separation of the four ions is possible for all but the shortest capillary. The separation is achieved in $30 \mathrm{~s}$ in the longest capillary and in about $10 \mathrm{~s}$ in the shortest capillary. Two features are apparent from the electro-

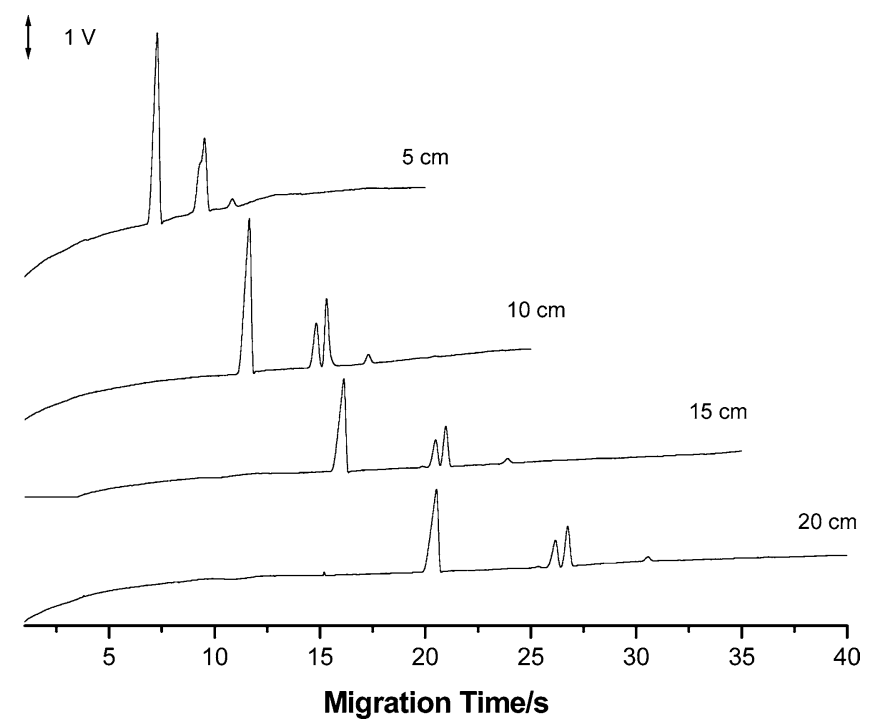

Fig. 2 Electropherograms for a test mixture of four inorganic cations in short capillaries of different effective lengths to the detector: $\mathrm{K}^{+}, \mathrm{Na}^{+}, \mathrm{Mg}^{2+}$, and $\mathrm{Li}^{+}$in this respective order of elution, all at $0.1 \mathrm{mM}$. Buffer $20 \mathrm{mM} \mathrm{MES/His,} \mathrm{pH} 6.1$; field strength $+850 \mathrm{~V} \mathrm{~cm}^{-1}$; capillary i.d. $50 \mu \mathrm{m}$; injection $4 \mathrm{~s}$ at $5 \mathrm{kV}$ pherograms. Firstly, a slight drift of the baseline towards higher conductivities is evident in all the electropherograms of Fig. 2, and the more pronounced the drift the shorter the capillary. The drift was also found to be stronger for higher applied field strengths and also to be strongly dependent on the capillary diameter as it was more pronounced with capillaries of $75-\mu \mathrm{m}$ i.d. The reason for this is not clear; it might be related to Joule heating or to some electrical charging phenomenon. In any case, it was found to be negligible for capillaries with $25-\mu \mathrm{m}$ internal diameters. It was furthermore found that the capillary diameter had almost no bearing on the detection limit. For potassium, for example, the detection limits for capillaries of $25-, 50-$, and $75-\mu \mathrm{m}$ i.d. were all found to be between 0.5 and $1 \mu \mathrm{M}$. Note that this is in strong contrast to optical detection methods. The special behavior in conductometric detection can be rationalized by considering that the change in diameter not only leads to a change in signal strength for the analyte but also for the background signal due to the buffer ions.

The second feature apparent from the electropherograms of Fig. 2 is the loss of resolution for the second and the third peaks for the shorter capillaries with the shorter analysis times. This pattern is of course expected as the peak separation depends on the length of time the analyte species are exposed to the field. In order to achieve fast analysis times in short channels any efforts to optimize separation must tackle the causes of band broadening. The main contribution for small ions such as those used here is generally diffusion, but other causes are wall interactions, electrodispersion (caused by differences in mobility between analyte ions and buffer ions leading to fronting and tailing of peaks), and Joule heating. It is also obvious from the traces of Fig. 2 that these mechanisms are significant on the short time scale of these separations as the peaks are smaller and broader for the longer separations.

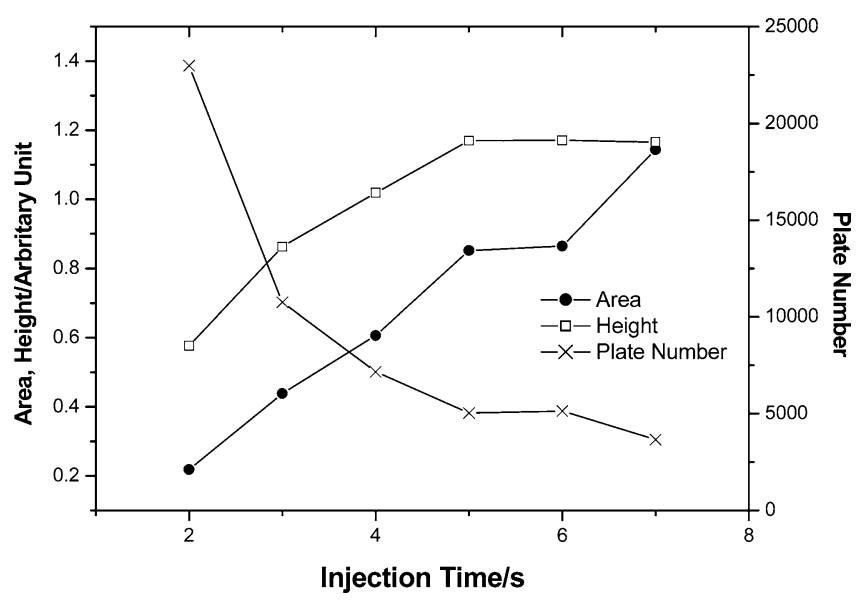

Fig. 3 Effect of injection time (at $5 \mathrm{kV}$ ) on peak efficiency, peak height, and peak area. $\mathrm{K}^{+} 0.1 \mathrm{mM}$; buffer $20 \mathrm{mM} \mathrm{MES} / \mathrm{His}$, $\mathrm{pH}$ 6.1; effective capillary length to detector $20 \mathrm{~cm}$ (total $35 \mathrm{~cm}$ ); field strength $+850 \mathrm{~V} \mathrm{~cm}^{-1}$; capillary i.d. $25 \mu \mathrm{m}$ 
A detailed quantitative elucidation and theoretical modeling of all overlapping causes of band broadening is very difficult to achieve, in particular when considering that extracolumn band-broadening effects due the injection process and the presence of a small residual hydrodynamic flow can also be expected to play a role. The study was therefore limited to an optimization of the parameters which can be readily changed with the aim of achieving best performance in the application of the system. An important factor is of course the size of the sample plug introduced and this indeed has a very pronounced effect on the efficiency as illustrated by the plot of plate numbers for the peak of potassium against injection time shown in Fig. 3. On the other hand, peak height and area are, of course, also dependent on the amount injected, and short injections will lead to a low sensitivity and hence higher limits of detection. It is also interesting to note that beyond an injection time of $5 \mathrm{~s}$, the peak height is constant, indicating that any effects leading to dilution do not reach the center of the sample plug in this case. Note that the size of the detection volume also has an effect on the separation efficiency; an enlargement of the electrode gap beyond the $1 \mathrm{~mm}$ employed for these measurements led to lower plate numbers. Smaller gaps, on the other hand, are not readily possible with the cell employed. The results also clearly demonstrate that better results in terms of efficiency could be obtained with a more sensitive detection method (such as fluorescence) as then the injected amount could be further reduced while still achieving adequate detection limits. In practice a compromise which satisfies the application at hand has to be made between separation efficiency and the sensitivity as determined by the detector employed.

It was found that the peak efficiency in terms of plate numbers was also strongly dependent on the type of ion. For example with a capillary of 50- $\mu \mathrm{m}$ diameter and 20$\mathrm{cm}$ effective length, plate numbers of $15,700,62,500$, 65,700 , and 86,000 were determined for $\mathrm{K}^{+}, \mathrm{Na}^{+}$, $\mathrm{Mg}^{2+}$, and $\mathrm{Li}^{+}$, respectively. A close examination of Fig. 2 also reveals fronting for the earlier species but none for $\mathrm{Li}^{+}$, the last peak. Evidently, electrodispersion, due to a non-perfect match with the mobility of the buffer cation, is an important contributor to band broadening for these ions. This is perhaps exacerbated in conductivity detection as the buffer has to be of low conductivity to give a good signal-to-noise ratio. The MES/His buffer solution employed has been used frequently for conductivity detection of small ions and represents a good compromise for good detection sensitivity and limited electrodispersion so that further optimization in this regard appears not readily possible. An increase in the electric field strength (for constant effective separation distances) was found to show a tendency to higher plate numbers, but the overall effect was slight. Presumably any reduction in band-broadening effects for the higher field strengths are offset by the shorter period of time available for separation. However, the capillary diameter is important. For $75-\mu \mathrm{m}$ capillaries the plate numbers are consistently lower then for capillaries of $50 \mu \mathrm{m}$ and $25 \mu \mathrm{m}$ which in turn showed no systematic difference between them. For $\mathrm{Li}^{+}$, for example, a plate number of 9,200 was found (for a field strength of $600 \mathrm{~V} \mathrm{~cm}^{-1}, 20$-cm effective capillary length, and an injection time of $4 \mathrm{~s}$ for $5 \mathrm{kV}$ ), but in 50 - and 25$\mu \mathrm{m}$ capillaries these were determined to be as high as 69,600 and 64,300 , respectively. This tendency coincides with the preference for narrow diameters to reduce baseline drifts as discussed above.

The highest plate number of approximately 90,000 was thus found for $\mathrm{Li}^{+}$using a capillary of $50-\mu \mathrm{m}$ i.d. and a field strength of $800 \mathrm{~V} \mathrm{~cm}^{-1}$ for a capillary of 20 -
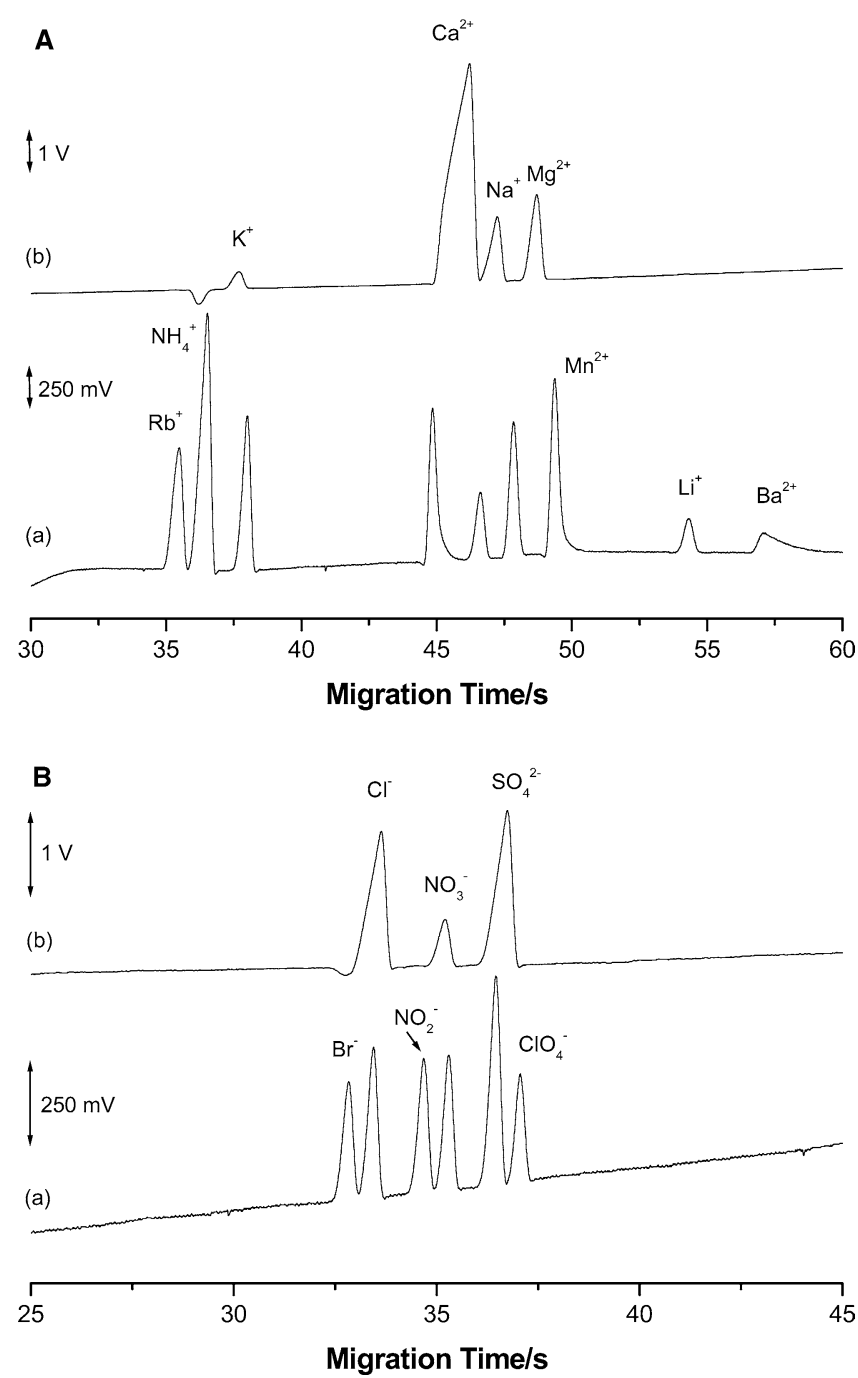

Fig. 4 a Electropherograms for a standard mixture of cations $(a)$ and of a sample of Rhine water $(b)$. Concentrations in $a 0.1 \mathrm{mM}$; buffer $20 \mathrm{mM}$ MES/His, $1 \mathrm{mM}$ 18-crown-6, pH 6.1; effective capillary length $23 \mathrm{~cm}$ (total $38 \mathrm{~cm}$ ); field strength $+600 \mathrm{~V} \mathrm{~cm}^{-1}$; capillary i.d. $50 \mu \mathrm{m}$; injection $3 \mathrm{~s}$ at $5 \mathrm{kV}$. b Electropherograms of a standard mixture of anions $(a)$ and of a sample of Rhine water $(b)$. Concentrations in a $0.1 \mathrm{mM}$; buffer $20 \mathrm{mM}$ MES/His, $0.00015 \%$ hexadimethrine bromide; effective capillary length $25 \mathrm{~cm}$ (total $45 \mathrm{~cm}$ ); field strength $-670 \mathrm{~V} \mathrm{~cm}^{-1}$; capillary i.d. $50 \mu \mathrm{m}$; injection $3 \mathrm{~s}$ at $-5 \mathrm{kV}$ 
$\mathrm{cm}$ effective length. For $10-$ and 5 -cm effective lengths the plate numbers obtained for this ion were 34,000 and 12,800 , respectively. Incidentally, the separation efficiencies and the analyses times achieved with the system reported herein compare well to results obtained on electrophoresis chips. Zhang and Manz [26] obtained plate numbers between 5,000 and 7,000 for the separation of the small ions fluorescein and fluorescein-5-isothiocyanate in a $2.8-\mathrm{cm}$ channel in a study on the effects of injection parameters. Higher plate numbers have been reported, for example, Obeid et al.[27] detected DNA fragments in a $6-\mathrm{cm}$ separation channel with plate numbers of 70,000; however, these have been achieved with large molecules, for which diffusional band broadening is minimal. Tanyanyiwa et al. [12] have investigated the determination of different kinds of small organic ions in chips with channels of $8-\mathrm{cm}$ effective length using contactless conductivity detection. Plate numbers between 2,000 and 4,200 were obtained.

The application of the system with optimized conditions to the determination of the major alkali and alkaline earth metal ions and ammonium in water samples is illustrated in Fig. 4. Clearly baseline separation can be achieved in less than $1 \mathrm{~min}$ for these ions. The same applies to the inorganic anions which can be separated in less than $40 \mathrm{~s}$, as is also shown in Fig. 4. These ions could also be quantified successfully with detection limits of $1 \mu \mathrm{M}$.

Contactless conductivity detection is not restricted to inorganic species. The fast separation of five carbohydrates is shown in Fig. 5. The detection of these species in electrophoresis, as well as in HPLC, is generally difficult as they are not UV-absorbent; conductometric detection is therefore an interesting proposition. Note that a highly alkaline buffer is necessary to cause

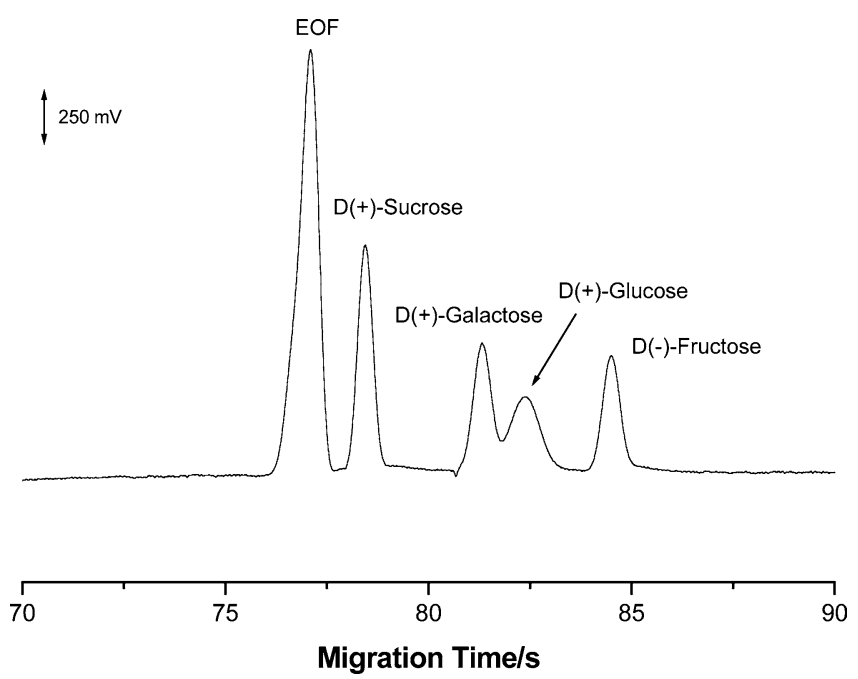

Fig. 5 Electropherogram for a mixture of carbohydrates (all $1 \mathrm{mM}$ ). Buffer $10 \mathrm{mM} \mathrm{NaOH}, 4.5 \mathrm{mM} \mathrm{Na} \mathrm{HPO}_{4}, 0.00015 \%$ hexadimethrine bromide, $\mathrm{pH}>12$; effective capillary length to detector $20 \mathrm{~cm}$ (total $35 \mathrm{~cm}$ ); field strength $+570 \mathrm{~V} \mathrm{~cm}^{-1}$; capillary i.d. $15 \mu \mathrm{m}$; injection $2 \mathrm{~s}$ at $-7 \mathrm{kV}$

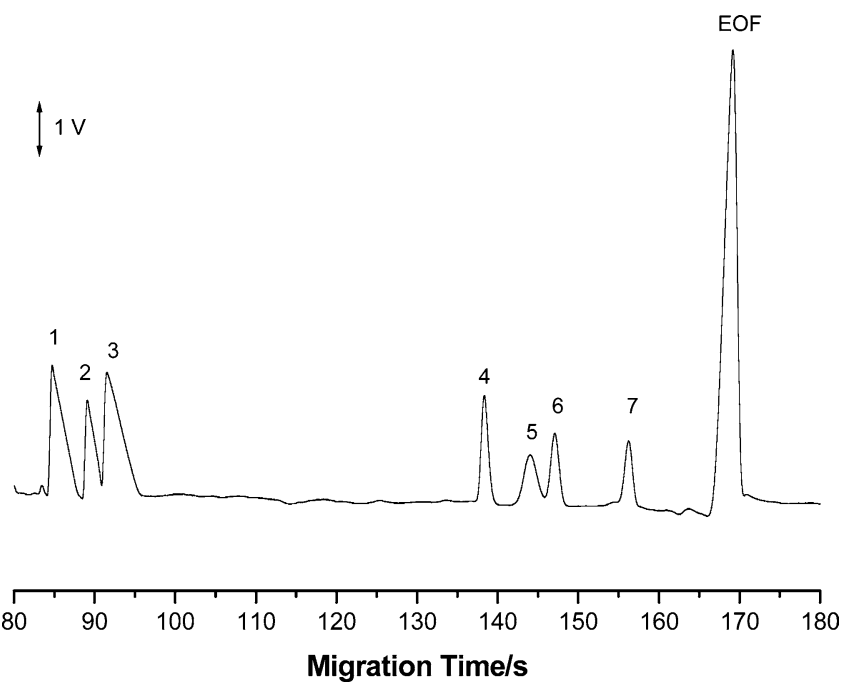

Fig. 6 Electropherogram for a mixture of amino acids and carbohydrates: 1 threonine, 2 methionine, 3 leucine, 4 fructose, 5 glucose, 6 galactose, 7 sucrose, all $1 \mathrm{mM}$; buffer $10 \mathrm{mM} \mathrm{NaOH}$, $4.5 \mathrm{mM} \mathrm{Na} \mathrm{HPO}_{4}, 1 \mathrm{mM} \mathrm{CTAB}, \mathrm{pH}>12$; effective capillary length to detector $25 \mathrm{~cm}$ (total $40 \mathrm{~cm}$ ); field strength $+500 \mathrm{~V} \mathrm{~cm}^{-1}$; capillary i.d. $15 \mu \mathrm{m}$; injection $2 \mathrm{~s}$ at $20 \mathrm{kV}$

ionization of the carbohydrates. The buffer solution was frequently replaced to preclude any effects which might arise from the absorption of $\mathrm{CO}_{2}$ from air. Joule heating caused by this buffer of relatively high conductivity is not significant with the small internal capillary diameter of $15 \mu \mathrm{m}$ employed. The high background conductivity also leads to negative-going deviations from the baseline, which is however not a problem. The peaks in Fig. 5 have been plotted in the conventional mode. Another group of important analytes which mostly cannot be detected by direct UV absorption are the

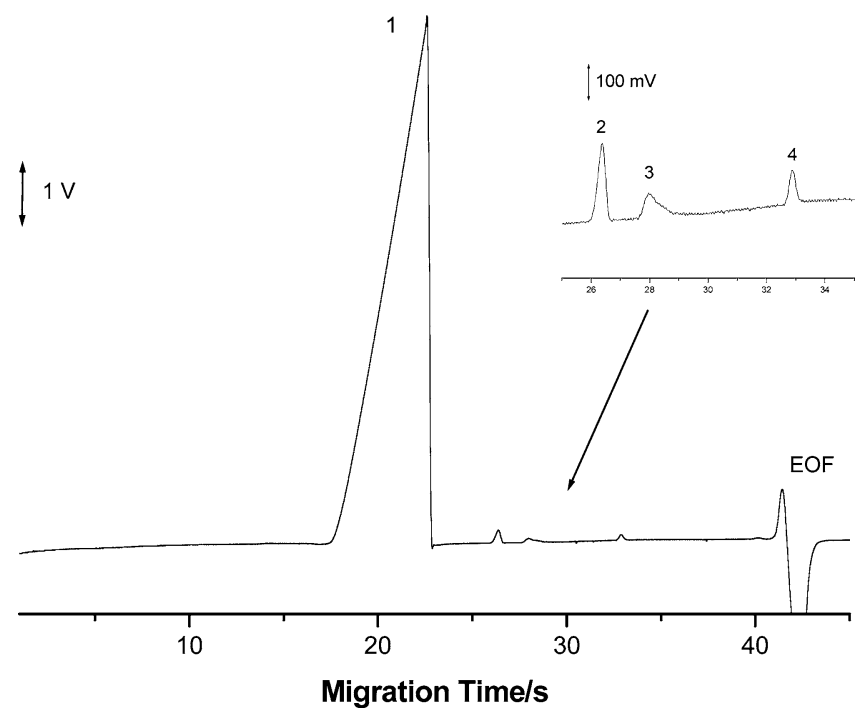

Fig. 7 Electropherogram for a pharmaceutical preparation: 1 sodium, $20.16 \mathrm{mM}$ ephedrine, $30.06 \mathrm{mM}$ doxylamine, 4 $0.13 \mathrm{mM}$ dextomethorphane; buffer $20 \mathrm{mM}$ MES/His, pH 6.1; effective capillary length to detector $15 \mathrm{~cm}$ (total $35 \mathrm{~cm}$ ); field strength $+850 \mathrm{~V} \mathrm{~cm}^{-1}$; capillary i.d. $25 \mu \mathrm{m}$; injection $7 \mathrm{~s}$ at $10 \mathrm{kV}$ 
amino acids. These may be determined as cations at strongly acidic $\mathrm{pH}$ values or as anions at high $\mathrm{pH}$ [15]. The basic conditions suitable for the analysis of the carbohydrates are also suitable for the amino acids such that both classes of analytes may also be determined in combination: this application is illustrated in Fig. 6 for a model mixture of three amino acids and four sugars.

The concurrent detection of inorganic and organic compounds in sample of pharmaceutical preparation is shown in Fig. 7. The formulation contains three active ingredients, ephedrine, doxylamine, and dextomethorphane, which are amine bases and cationic at neutral $\mathrm{pH}$ value, and a high concentration of sodium ions. The four compounds could easily be baseline-separated in less than $40 \mathrm{~s}$. Although the concentration of sodium in the sample is not known, the wide dynamic range of the conductometric detection method is nicely illustrated by the figure and its inset.

\section{Conclusion}

Rapid separations with a performance, in terms of analysis time and separation efficiency, similar to that reported for electrophoretic chip devices have been obtained with short conventional capillaries. The arrangement has the advantage of higher flexibility. Generally three parameters may be varied to achieve optimum compromise conditions in terms of sensitivity and analysis time, namely, separation voltage, separation length, and injection volume. With our set-up, in contrast to electrophoresis chips, the channel length in particular can be extended and optimized should it not be adequate for the task at hand, as the detector can easily be moved along the capillary. Note, however, that this is not possible with commercial capillary electrophoresis instruments because of constraints imposed by the design. With contactless conductivity detection this adjustment can be made in an instant, since it is not necessary to remove the polyimide coating on the fused silica capillary to create an optical window as for UV absorption and fluorescence detection. Furthermore, higher sensitivities are possible with contactless conductivity detection on conventional capillaries than on chips because of limitations on electrode placement on the latter.
Acknowledgements The authors are grateful to the Swiss National Science Foundation for financial support (grant number 200067830).

\section{References}

1. Zemann AJ, Schnell E, Volgger D, Bonn GK (1998) Anal Chem 70:563-567

2. Fracassi da Silva JA, do Lago CL (1998) Anal Chem 70:43394343

3. Tanyanyiwa J, Leuthardt S, Hauser PC (2002) Electrophoresis 23:3659-3666

4. Zemann AJ (2003) Electrophoresis 24:2125-2137

5. Zemann AJ (2001) Trends Anal Chem 20:346-354

6. Tanyanyiwa J, Hauser PC (2002) Electrophoresis 23:3781-3786

7. Mayrhofer K, Zemann AJ, Schnell E, Bonn GK (1999) Anal Chem 71:3828-3833

8. Tanyanyiwa J, Leuthardt S, Hauser PC (2002) J Chromatogr A 978:205-211

9. Kuban P, Karlberg B, Kuban V (2002) J Chromatogr A 964:227-241

10. Laugere F, Guijt RM, Bastemeijer J, van der Steen G, Berthold A, Baltussen E, Sarro P, van Dedem GWK, Vellekoop M, Bossche A (2003) Anal Chem 75:306-312

11. Tanyanyiwa J, Galliker B, Schwarz MA, Hauser PC (2002) Analyst 127:214-218

12. Tanyanyiwa J, Abad-Villar EM, Hauser PC (2004) Electrophoresis 25:903-908

13. Tanyanyiwa J, Hauser PC (2004) Electrophoresis 25:3010-3016

14. Coufal P, Zuska J, van de Goor T, Smith V, Gas B (2003) Electrophoresis 24:671-677

15. Tanyanyiwa J, Schweizer K, Hauser PC (2003) Electrophoresis 24:2119-2124

16. Carvalho AZ, da Silva JAF, do Lago CL (2003) Electrophoresis $24: 2138-2143$

17. Tanyanyiwa J, Hauser PC (2002) Anal Chem 74:6378-6382

18. Zemann AJ (1997) J Chromatogr A 787:243-251

19. Yang WP, O'Flaherty B, Cholli AL (2001) J Environ Sci Health A 36:1271-1285

20. Yang WP, Zhang ZJ (2002) Int J Environ Anal Chem 82:353360

21. Yang WP, Zhang ZJ (2003) Anal Lett 36:465-477

22. Bjørnsdottir I, Hansen SH (1999) J Biochem Biophys Methods 38:155-161

23. Hyötyläinen T, Siren H, Riekkola ML (1996) J Chromatogr A 735:439-447

24. Rodriguez I, Zhang Y, Lee HK, Li SFY (1997) J Chromatogr A 781:287-293

25. Hooker TF, Jorgenson JW (1997) Anal Chem 69:4134-4142

26. Zhang C-X, Manz A (2001) Anal Chem 73:2656-2662

27. Obeid PJ, Christopoulos TK, Ioannou PC (2004) Electrophoresis 25:922-930 